\title{
A novel CSI-based fingerprinting for localization with a single AP
}

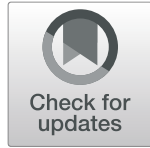

Lei Zhang ${ }^{1,2,3}$, Enjie Ding ${ }^{1,2,3}$, Yanjun $\mathrm{Hu}^{1,2,3^{*}}$ and Yafeng Liu ${ }^{1,2,3}$

\begin{abstract}
WiFi-based indoor localization techniques are critical for location-based services. Among them, fingerprint-based method gains considerable interest due to its high accuracy and low equipment requirement. One of the major challenges faced by fingerprint-based position system is that in some places there are not enough access points (AP) to provide features for accurate location. To address that, we propose a novel fingerprint-based system using only a single AP. We propose a novel phase decomposition method to obtain the phase of multipath provided by a AP and use the decomposed phase as a fingerprint after the feature exaction by principal component analysis (PCA). Performance in the laboratory, meeting room, and corridor is investigated, and our system is also compared with a RSSI-based and a CSI-based fingerprint localization system. As the experimental results suggest, the minimum mean distance error is $0.6 \mathrm{~m}$ in the laboratory, $0.45 \mathrm{~m}$ in the meeting room, and $1.08 \mathrm{~m}$ in the corridor, outperforming the other two systems.
\end{abstract}

Keywords: WiFi network, Indoor localization, Channel state information, Phase decomposition, MIMO-OFDM

\section{Introduction}

In outdoor environments, GPS-based localization techniques make our lives more convenient in many aspects $[1,2]$. Since the GPS signals cannot penetrate efficiently indoors to localize objects [3], some other techniques are used to replace GPS to provide accurate location information $[4,5]$ in indoor environment. Among the indoor localization techniques, the WiFi-based localization techniques have become one of research hotspots due to the ubiquitous WiFi infrastructure. However, in some occasions, there may not be enough APs to implement the location methods [6-8], hence it is necessary to study the location method based on single AP.

Since the received signal strength indication (RSSI) is capable of reflecting the distance between the AP and the node, traditional WiFi-based models use RSSI as a metric. A RSSI-based model or fingerprint can be built, and examples for RSSI-based localization systems are DWELT system [9] as well as Horus system [10]. Nevertheless, RSSI fluctuates significantly in some

\footnotetext{
* Correspondence: 303363209@qq.com

${ }^{1}$ School of Information and Control Engineering, China University of Mining and Technology, Xuzhou 221000, China

${ }^{2}$ IoT Perception Mine Research Center, China University of Mining and

Technology, Xuzhou 221000, China

Full list of author information is available at the end of the article
}

scenarios as being susceptible to multipath effect [11]. The positioning accuracy of most systems ranges from 3 to $10 \mathrm{~m}$ [12]. To overcome the fluctuation of RSSI, Hossain et al. proposed SSD [13] that localizes nodes using the signal strength difference. However, RSSI is limited as it fails to reflect the spatial, temporal, and environmental characteristics.

Signals transmitted in WiFi networks exploit orthogonal frequency division multiplexing (OFDM) [14], which are transmitted and received by multiple subcarriers. The received signal impacted by scattering, fading, and power loss with distance can be represented by the physical layer information known as channel state information (CSI). By modifying driver firmware, CSI can be obtained using Intel 5300 or Atheros 9390 cards $[15,16]$. Due to multipath, subcarriers will propagate along different paths, which makes the amplitude and phase of each subcarrier different from each other.

It is witnessed in recent years that CSI-based techniques have been widely adopted in localization. In comparison with coarse-grained RSSI, CSI is a fine-grained indicator containing amplitude and phase information during signal propagation [17-20]. Moreover, similar to RSSI-based techniques, model-based techniques are also used. LiFS using the subcarriers with minimal 
interference by multipath is designed by Wang et al. [21] for accurate localization of fingerprint. The combination of the amplitude with spatial diversity is used by $\mathrm{Wu}$ et al. [22] for indoor location. Furthermore, to achieve higher accuracy by obtaining high data dimension, FIFS [23] and CSI-MIMO [20] systems, both aggregate amplitude and phase information of all subcarriers of all antennas for indoor location fingerprinting. Angle of arrival (AOA) estimation is also applied in CSI-based localization such as ArrayTrack [24] whose key method is that make the number of antennas greater than the number of paths, and then deduces AOAs using MUSIC algorithm. By increasing the number of APs or the number of antennas, CSI-MIMO and ArrayTrack achieve high positioning accuracy, respectively. In practice, most of today's WiFi networks deployment is primarily for communications rather than for positioning. Thus, there may be insufficient APs to provide CSIs to generate a fingerprint, and increasing the number of antennas results in additional hardware costs which may not be available in practical applications as well.

In the present study, we try to advance CSI-based indoor localization to achieve centimeter-level localization resolution only by using a single commercial AP under the phase of multipath offered by CSI of subcarriers using multiple input multiple output (MIMO) to derive the location signatures.

The main contributions of this paper are as follows:

1. We design a method to obtain phases of multipath by decomposing construction CSI matrix of a single AP.

2. We propose to use phase information of multipath for indoor fingerprinting. To the best of our knowledge, this is the first work to leverage phase information of a single AP for indoor fingerprinting.

3. We analyze the effectiveness of the proposed location signature using deterministic k-nearest neighbor $(\mathrm{KNN})$, probabilistic Bayes algorithms, and support vector machine (SVM) in the experimental scenarios.

4. We investigate impact factors on the localization accuracy of our system such as the number of decomposition path, the size of training and estimation samples, the number of APs, and the size of the cell.

5. We evaluate the performance of our system with RSSI-based and CSI-based localization system.

The rest of this study is structured as follows. The related work is briefly presented is in Section 2. Section 3 introduces preliminary study including CSI. In Section 4, our proposed localization system is detailed. The evaluation results of our system are discussed in Section 5. The conclusion is drawn and the future research is directed in the final Section.

\section{Related work}

Increasing demand of location-based service (LBS) draws a great deal of attention to WiFi-based localization in indoor environments. We catalog this into two classes, device-free localization and device-based localization.

\subsection{Device-free localization}

As it is not convenient for a subject to carry a device, Jie et al. [25] implement a localization system with non-equipped entity. Some other systems [26-30] have been proposed which constitute fingerprint-based or model-based solutions. Model-based algorithms, which do not require any laborious effort to build and maintain a radio map, estimate the distance from the object to an AP by using statistical models. LiFS [21] achieves the accuracy of about $1 \mathrm{~m}$ in indoor environments by using selected subcarriers of CSI to build a model. Due to the changes of direction, reflecting, or scattering signals, it is hard to obtain an accurate relationship between the signal and the location. Fingerprint-based algorithms, however, need no prior knowledge of the relationship between the distance and signals, gain much attention in the localization systems. General fingerprint-based technique has two phases named the offline and localization phase. Offline phase is to collect signals for generating fingerprints from every spot of an interested area to build a radio map. During the localization phase, observed fingerprint is matched against the radio-map by using matching algorithms. Zhou et al. [31] establish the nonlinear relationship between CSI fingerprints during the offline phase and estimate locations through SVM regression. Unlike fingerprinting localization, MaTrack [32] uses Dynamic-MUSIC method to identify the angles for localization through detecting the subtle reflection signals from the human body.

\subsection{Device-based localization}

Due to security or commercial reasons, in some scenarios, we not only need the position but also the identity information of a person in which the device-free localization system is not suitable. Device-based localization system can provide identity information as human carrying specialized equipment. Compared to using RSSI of WiFi for localization such as Horus [10] and RADAR [33], CSI-based localization systems provide higher accuracy. PILA [18] designs an algorithm to identify direct path from multipath and uses MUSIC algorithm to jointly estimate the AOA and time of arrival (TOA) of each path. PhaseFi [34] implements a scheme in which the calibrated phase information is extracted for fingerprinting in the offline stage and a deep learning approach is used for location estimation in the localization stage. As the bandwidth of WiFi is limited, 
ToneTrack [35] increases the effective bandwidth to obtain the TOA of APs by leveraging the frequency-agile technique. Similar to ToneTrack, CHRONOS [36] uses Chinese remainder theorem to measure the time of fly (TOF) across different channels and then estimate distance between devices and AP through leveraging the TOF measurements. In addition to considering positioning accuracy, positioning efficiency is also of great importance to localization systems. For instance, CRIL [37] accounts for dynamic environments introduces coupled RSSI and INS localization system to improve the efficiency of location estimation.

\section{Preliminaries}

\section{$3.1 \mathrm{CSI}$}

In wireless communication systems, CSI reveals the channel response properties of transmission links, which includes the combined effect of scattering, fading, and power decay with distance. In most WiFi networks, multiple input multiple output (MIMO) and OFDM technologies are employed to transmit data more effectively. In these networks, CSI represents the subcarriers properties of each transmitting and receiving antennas. Halperin et al. [38] released a tool by which both the amplitude and phase measurements of each MIMO-OFDM subcarrier can be aggregated from Intel's IWL 5300.

According to the bandwidth of $\mathrm{WiFi}$, it is considered as a narrowband flat fading channel, and the channel model is defined as follows:

$$
y=\operatorname{CSI} x+n
$$

where $y$ and $x$ denote the received and transmitted signal vectors, respectively, CSI denotes the channel frequency response, and $n$ is the additive white Gaussian noise.

In $802.11 \mathrm{n}$ standard, 56 or 114 subcarriers are used for data transmission in the OFDM system. Each antenna of the IWL 5300 exports 30 out of all subcarriers and the index of subcarriers varies with bandwidth and grouping $\mathrm{Ng}$. The $\mathrm{CSI}_{k}$ denotes the channel frequency response of the subcarrier $k$ shown as follows:

$$
C S I_{k}=\sum_{l=1}^{L} \alpha_{l} e^{-j 2 \pi\left(f_{0}+k \Delta f\right) \tau_{l}}
$$

where $\alpha_{l}$ and $\tau_{l}$ denote the signal magnitude and the TOF of path $l$, respectively. $f_{0}$ is the central frequency, $\Delta f$ is the frequency interval of adjacent subcarriers, and the 30 subcarriers are written as follows:

$$
\mathbf{C S I}=\left[\mathrm{CSI}_{1}, \mathrm{CSI}_{2}, \ldots \mathrm{CSI}_{30}\right]
$$

\subsection{MIMO}

MIMO technology using multiple transmitting and receiving antennas greatly increases the capacity and spectrum utilization of the communication systems without increasing the bandwidth. Due to the multipath effects, it is difficult to achieve high-speed data transmission in cluttered indoor environments [39]. However, in MIMO systems, multipath effects can serve as a favorable factor, as the signal among different transmitting antennas uses multipath propagation. Moreover, if the antennas of both transmitting and receiving are independent, the data transmission will be improved in these parallel spatial channels created by the MIMO system. Set $t$ and $r$ as the number of transmitting and receiving antennas, respectively. Each transmitting and receiving antenna pair is comprised of a data-stream. The CSIs data of all antennas are defined as follows:

$$
\mathbb{C S I}=\left[\begin{array}{cccc}
\mathbf{C S I}_{11} & \mathrm{CSI}_{12} & \ldots & \mathrm{CSI}_{1 t} \\
\mathbf{C S I}_{21} & \mathrm{CSI}_{22} & \ldots & \mathbf{C S I}_{2 t} \\
\ldots & \ldots & \ldots & \ldots \\
\mathrm{CSI}_{r 1} & \mathbf{C S I} & \ldots & \mathbf{C S I}_{r t}
\end{array}\right]
$$

where the $\mathbf{C S I}_{r t}$ is a vector denoting the CSI of 30 subcarriers between the transmitting antenna $t$ and receiving antenna $r$.

\section{Motivation and localization system design}

In this section, we investigate the challenges posed by CSI phase measurements to improve the location estimation. In accordance with (2), CSI is the superposition of multipath signals. If there is only a direct path, phase can serve as a fingerprint for localization. However, in practical indoor environments, indoor propagation is dominated by severe multipath as shown in Fig. 1, and raw phase information is not suitable for generating fingerprints. To use phase as a fingerprint, we design a method to obtain the phase from multipath.

The architecture of the system is designed with four key constituent blocks, as shown in Fig. 2. We will describe each block in detail in the following subsections.

\subsection{Overview}

The system is built on a commercial off-the-self WiFi infrastructure without additional hardware. It has two phases, training and estimation. The overview positioning process is as follows: 


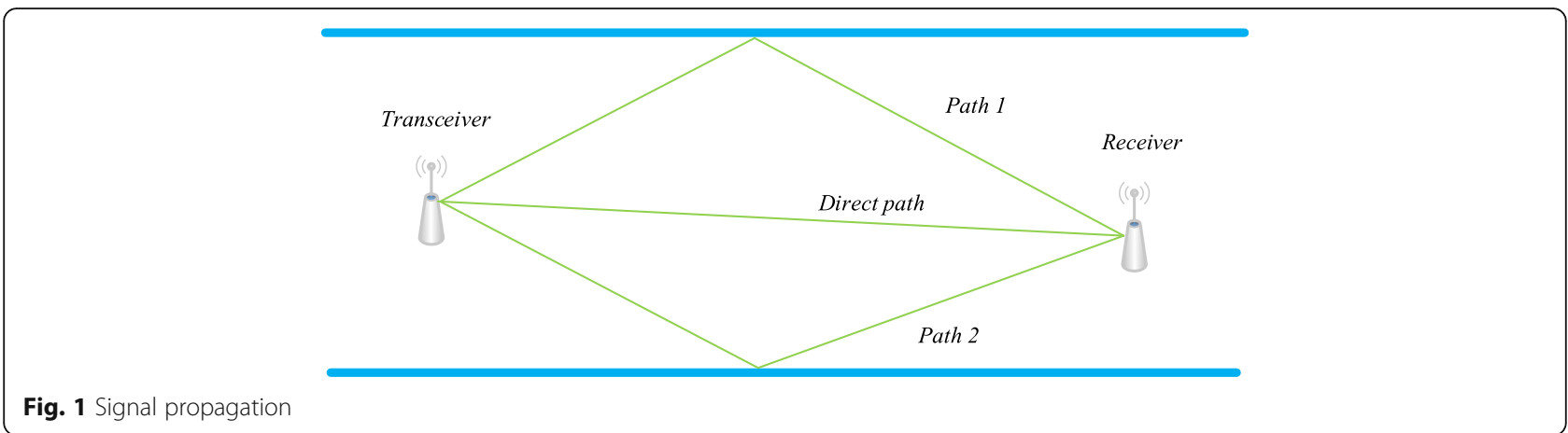

\subsubsection{Training phase}

In training phase, a mobile device is placed at a known location for the collection of CSI data. After outlier removal, a phase sanitation block is used to calibrate phase, and then the phase information of the multipath is obtained through a phase decomposition block, which is presented later in this section. A unique fingerprint of one location is generated using the feature extraction method based on dimensionality reduction. This work is repeated on each location until we store the whole fingerprint coupled with the coordinates in the fingerprint database.

\subsubsection{Estimation phase}

During the online phase, we randomly select some unknown locations to collect CSI data. Similar to the training phase, the collected CSI data is processed using the same method to generate a position fingerprint. A fingerprint matching algorithm is employed to compare it with the location fingerprint database to estimate the object location.

\subsection{Outlier removal}

As the outliers appear during our collection due to the indoor environmental noise, which is capable of affecting the localization system performance. Consequently, we implement Pauta Criterion [40] to remove these biased measurements, as shown in the following:

$$
\begin{aligned}
& V_{m}=\left|X_{m}-\bar{X}\right|>3 S \\
& S=\sqrt{\frac{\sum_{i=1}^{n}\left(X_{i}-\bar{X}\right)^{2}}{n-1}}
\end{aligned}
$$

where $X_{m}$ denotes the one of all the measurement, and $\bar{X}$ is the median. We declare $X_{m}$ as an outlier if it meets the condition according to (4). The CSIs of four subcarriers before and after performing outlier removal are shown in Fig. 3. It is observed that the Pauta Criterion works well to remove the significant outliers.

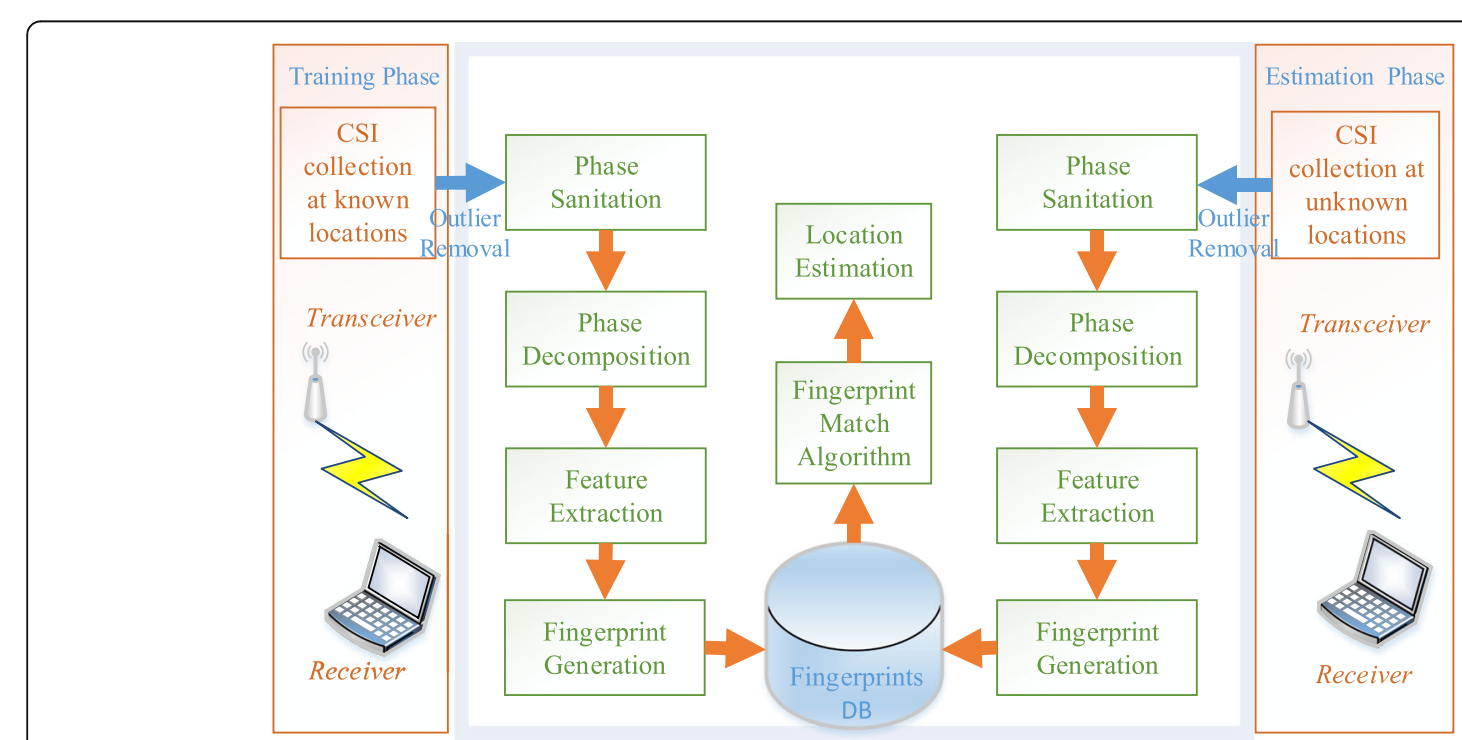

Fig. 2 System architecture 

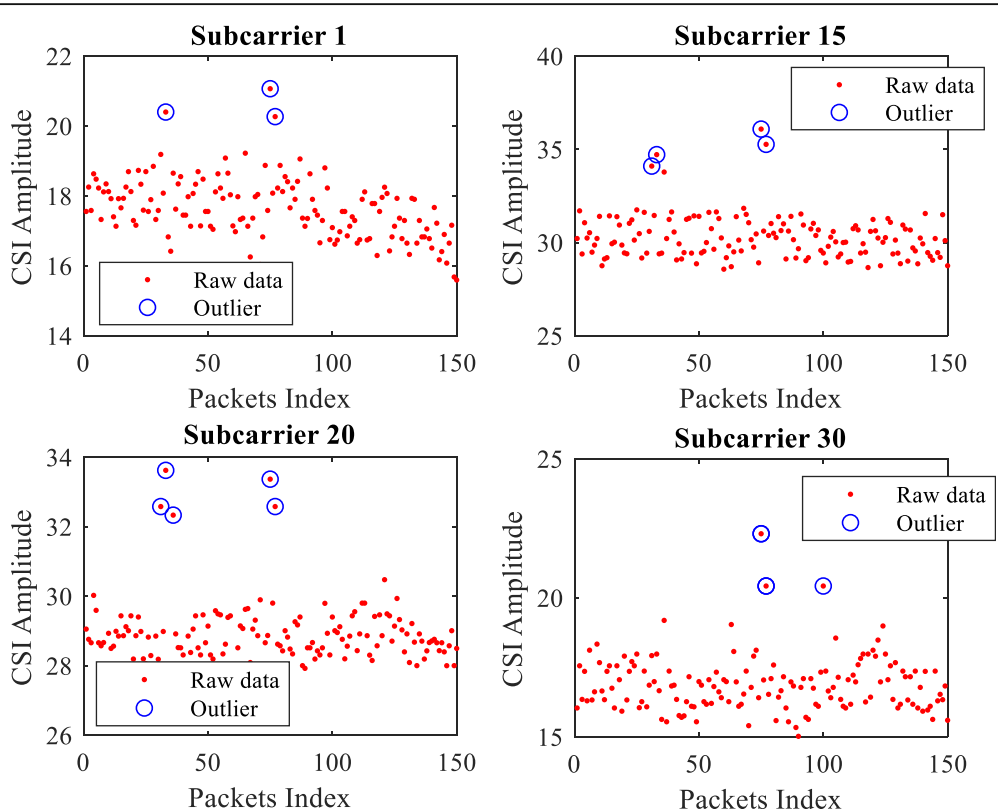

Fig. 3 Outlier removal. Each red point in this figure denotes the CSI amplitude of the subcarrier of each packet, and the blue circle is the outlier found by applying Pauta criterion

\subsection{Phase sanitization}

The hardware imperfection makes it impossible to gain the genuine CSI phase. Practically, there are two major causes of the measurement errors. One is the carrier frequency offset (CFO) as the center frequency is not synchronized between the transmitter and receiver. The sampling frequency offset (SFO) generated by non-synchronized clocks of ADC makes the measurement errors different for different subcarriers. The measured phase of subcarrier $k$ is written as follows:

$$
\angle \widehat{C S I}_{k}=\angle C S I_{k}+2 \pi \frac{n_{k}}{N} \Delta t_{k}+\beta+Z
$$

where the $\angle \widehat{\boldsymbol{C S I}}_{\boldsymbol{k}}$ and the $\angle C S I_{k}$ denote the measured and the genuine values of the subcarrier $k$, respectively. $n_{k}$ is the index of sub-carriers, and $\Delta t_{k}$ indicates the time offset due to SFO. $N$ is the FFT size from the IEEE 802.11n specification. $\beta$ is the unknown phase offset for CFO, and $Z$ is the measurement noise. It is unlikely to obtain the true phase information as the unknown $\Delta t_{k}$ and $\Delta t_{k}$. To eliminate phase offset $\Delta t_{k}$ and $\Delta t_{k}$, the linear transformation algorithm [41] is used to achieve this goal.

The offset $b$ and the slope $a$ are defined as follows:

$$
\begin{aligned}
& a=\frac{\angle \widehat{C S I}_{N}-\angle \widehat{C S I}_{1}}{n_{N}-n_{1}}=\frac{\angle C S I_{N}-\angle C S I_{1}}{n_{N}-n_{1}}-\frac{2 \pi}{N} \Delta t \\
& b=\frac{1}{30} \sum_{i=1}^{30} \angle \widehat{C S I}_{k}=\frac{1}{30} \sum_{k=1}^{N} \angle C S I_{k}+\frac{2 \pi \Delta t}{30 N} \sum_{n_{k}=1}^{N} n_{k}+\beta+Z
\end{aligned}
$$

In the OFDM system, the symmetric frequency of the subcarriers leads $\sum_{n_{k}=1}^{N} n_{k}=0$. Then, the $b$ is written as follows:

$$
b=\frac{1}{30} \sum_{i=1}^{30} \angle \widehat{C S I}_{k}=\frac{1}{30} \sum_{k=1}^{N} \angle C S I_{k}+\beta+Z
$$

By subtracting the linear term $a n_{k}+b$ from the raw phase, we obtain the calibrated values as follows:

$$
\angle \widetilde{C S I}_{k}=\angle C S I_{k}-\frac{\angle C S I_{N}-\angle C S I_{1}}{n_{N}-n_{1}} n_{k}-\frac{1}{30} \sum_{k=1}^{N} \angle C S I_{k}
$$

The CSI phase after sanitation is shown in Fig. 4a. Calibrated phases are less fluctuant and more concentrated, in comparison with the raw phases which scatter randomly over many different angles. The calibrated phases for about 80 packets at 3 different locations are shown in Fig. 4b. As can be seen, the calibrated phases are stable at one given location and differing in different locations, suggesting that it can be very suitable for indoor fingerprint positioning.

\subsection{Phase decomposition}

Compared with RSSI, CSI is a fine-grained information, yet the CSI value of each subcarrier is also a superposition of some paths due to the bandwidth of WiFi network. In this part, we strive to acquire the phase information of as many paths as possible by designing a phase decomposition method.

Set $C S I_{0}$ as the subcarrier of index 0. According to 2), $C S I_{k}$ is a linear combination of the phase of each path and the phase of subcarrier 0 . To simplify the notations, $C S I_{k}$ is represented as follows: 

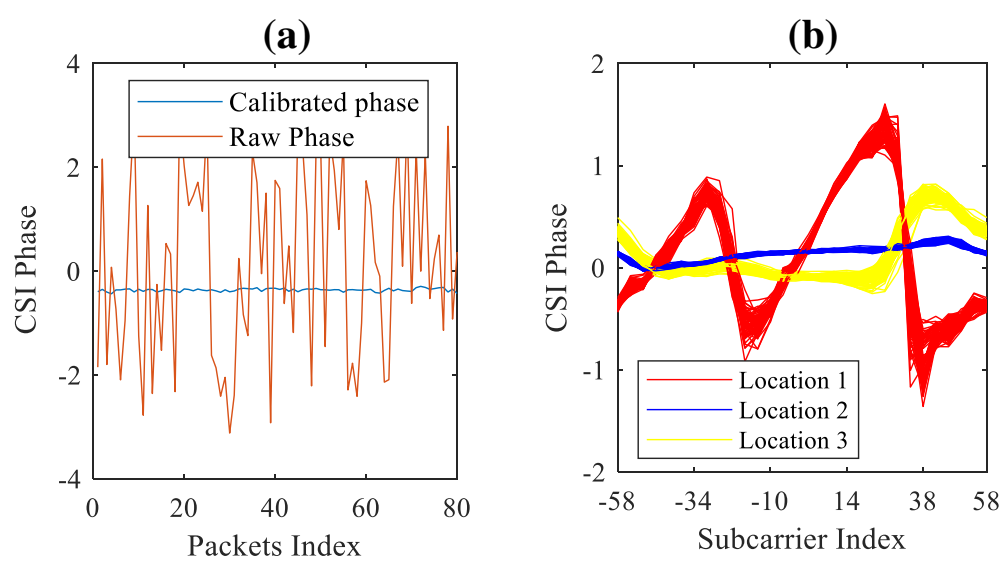

Fig. 4 a Raw phase vs. calibrated phase. The orange line in is the raw phase value of each packet and the blue line is presented as calibrated phase after linear transformation algorithm. b Calibrated phase at three different locations. Red, blue, and yellow line illustrate the calibrated phase of subcarriers at different locations, respectively

$$
C S I_{k}=\sum_{l=1}^{L} S_{0}^{l} \Delta_{k}^{l}
$$

For the path $l$ :

$$
\begin{gathered}
S_{0}^{l}=\alpha_{l} e^{-j 2 \pi f_{0} \tau_{l}} \\
\Delta_{k}^{l}=e^{-j k \Delta f \tau_{l}}
\end{gathered}
$$

In the $802.11 \mathrm{n}$ standard, when $\mathrm{BW}=40 \mathrm{MHz}$ and $\mathrm{Ng}$ $=4$ (grouping), the index of the sampled subcarriers ranges from -58 to 58 , in which the interval of subcarrier index is 4 . For the convenience of presentation, assuming that there are five paths here, and a Hankel matrix can be built:

$$
\mathbf{X}=\left[\begin{array}{lllll}
C S I_{-58} & C S I_{-54} & C S I_{-50} & C S I_{-46} & C S I_{-42} \\
C S I_{-54} & C S I_{-50} & C S I_{-46} & C S I_{-42} & C S I_{-38} \\
C S I_{-50} & C S I_{-46} & C S I_{-42} & C S I_{-38} & C S I_{-34} \\
C S I_{-46} & C S I_{-42} & C S I_{-38} & C S I_{-34} & C S I_{-30} \\
C S I_{-42} & C S I_{-38} & C S I_{-34} & C S I_{-30} & C S I_{-26}
\end{array}\right]
$$

Actually, $\mathbf{X}$ is written as follows:

$$
\mathbf{X}=\Delta \mathbf{S} \boldsymbol{\Delta}^{T}
$$

Where

$$
\Delta=\left[\begin{array}{ccccc}
\Delta_{-29}^{1} & \Delta_{-29}^{2} & \Delta_{-29}^{3} & \Delta_{-29}^{4} & \Delta_{-29}^{5} \\
\Delta_{-25}^{1} & \Delta_{-25}^{2} & \Delta_{-25}^{3} & \Delta_{-25}^{4} & \Delta_{-25}^{5} \\
\Delta_{-21}^{1} & \Delta_{-21}^{2} & \Delta_{-21}^{3} & \Delta_{-21}^{4} & \Delta_{-21}^{5} \\
\Delta_{-17}^{1} & \Delta_{-17}^{2} & \Delta_{-17}^{3} & \Delta_{-17}^{4} & \Delta_{-17}^{5} \\
\Delta_{-13}^{1} & \Delta_{-13}^{2} & \Delta_{-13}^{3} & \Delta_{-13}^{4} & \Delta_{-13}^{5}
\end{array}\right]
$$

And

$$
\mathbf{S}=\operatorname{diag}\left(S_{0}^{1}, S_{0}^{2}, S_{0}^{3}, S_{0}^{4}, S_{0}^{5}\right)
$$

We define $\Delta=\mathbf{V S}^{\prime}$.
Where

$$
\mathbf{V}=\left[\begin{array}{ccccc}
1 & 1 & 1 & 1 & 1 \\
\Delta_{4}^{1} & \Delta_{4}^{2} & \Delta_{4}^{3} & \Delta_{4}^{4} & \Delta_{4}^{5} \\
\Delta_{8}^{1} & \Delta_{8}^{2} & \Delta_{8}^{3} & \Delta_{8}^{4} & \Delta_{8}^{5} \\
\Delta_{12}^{1} & \Delta_{12}^{2} & \Delta_{12}^{3} & \Delta_{12}^{4} & \Delta_{12}^{5} \\
\Delta_{16}^{1} & \Delta_{16}^{2} & \Delta_{16}^{3} & \Delta_{16}^{4} & \Delta_{16}^{5}
\end{array}\right]
$$

And

$$
\mathbf{S}^{\prime}=\operatorname{diag}\left(S_{-29}^{1}, S_{-29}^{2}, S_{-29}^{3}, S_{-29}^{4}, S_{-29}^{5}\right)
$$

Thus, $\mathbf{X}=\left(\mathbf{V S}^{\prime}\right) \mathbf{S}\left(\mathbf{V S}^{\prime}\right)^{T}$ since $\mathbf{S}^{\prime}=\left(\mathbf{S}^{\prime}\right)^{T} \mathbf{X}$ is presented as follows:

$$
\mathbf{X}=\mathbf{V} \boldsymbol{\Sigma} \mathbf{V}^{T}
$$

It is easy to prove that

$$
\begin{aligned}
& \mathbf{V}=\left[\begin{array}{ccccc}
1 & 1 & 1 & 1 & 1 \\
\Delta_{4}^{1} & \Delta_{4}^{2} & \Delta_{4}^{3} & \Delta_{4}^{4} & \Delta_{4}^{5} \\
\Delta_{8}^{1} & \Delta_{8}^{2} & \Delta_{8}^{3} & \Delta_{8}^{4} & \Delta_{8}^{5} \\
\Delta_{12}^{1} & \Delta_{12}^{2} & \Delta_{12}^{3} & \Delta_{12}^{4} & \Delta_{12}^{5} \\
\Delta_{16}^{1} & \Delta_{16}^{2} & \Delta_{16}^{3} & \Delta_{16}^{4} & \Delta_{16}^{5}
\end{array}\right] \\
& =\left[\begin{array}{ccccc}
1 & 1 & 1 & 1 & 1 \\
\left(\Delta_{4}^{1}\right)^{1} & \left(\Delta_{4}^{2}\right)^{1} & \left(\Delta_{4}^{3}\right)^{1} & \left(\Delta_{4}^{4}\right)^{1} & \left(\Delta_{4}^{5}\right)^{1} \\
\left(\Delta_{4}^{1}\right)^{2} & \left(\Delta_{4}^{2}\right)^{2} & \left(\Delta_{4}^{3}\right)^{2} & \left(\Delta_{4}^{4}\right)^{2} & \left(\Delta_{4}^{5}\right)^{2} \\
\left(\Delta_{4}^{1}\right)^{3} & \left(\Delta_{4}^{2}\right)^{3} & \left(\Delta_{4}^{3}\right)^{3} & \left(\Delta_{4}^{4}\right)^{3} & \left(\Delta_{4}^{5}\right)^{3} \\
\left(\Delta_{4}^{1}\right)^{4} & \left(\Delta_{4}^{2}\right)^{4} & \left(\Delta_{4}^{3}\right)^{4} & \left(\Delta_{4}^{4}\right)^{4} & \left(\Delta_{4}^{5}\right)^{4}
\end{array}\right]
\end{aligned}
$$

V is a Vandermonde matrix [42]. Therefore, the problem arises to find a Vandermonde decomposition of $\mathbf{X}$. The decomposition algorithm [43] is shown as follows: 


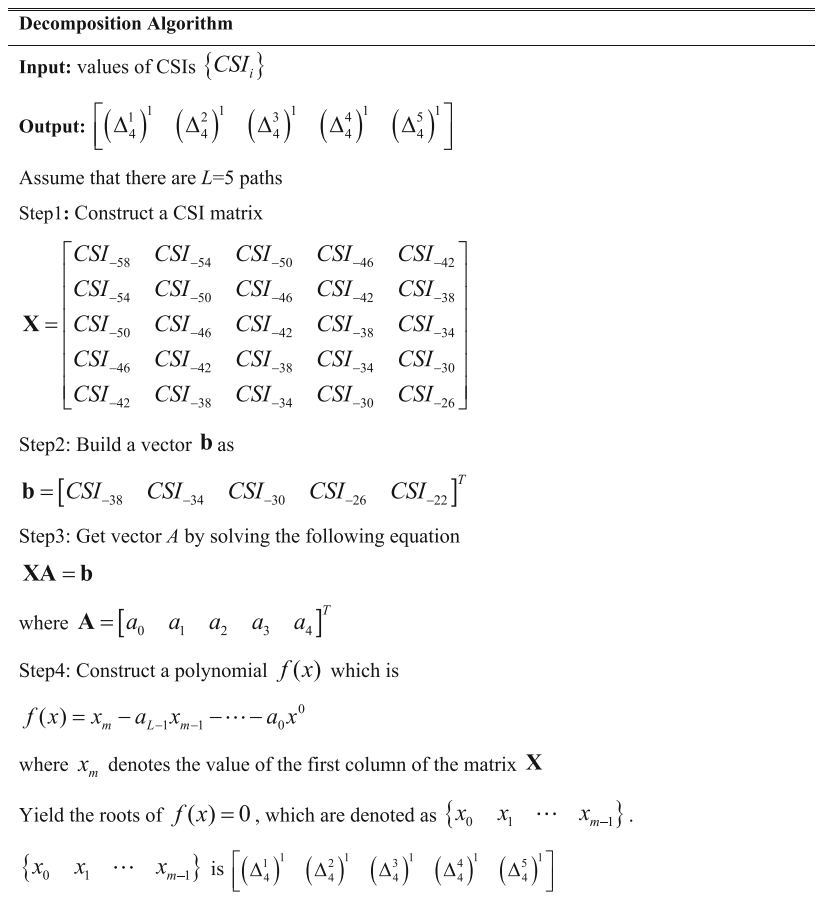

According to phase decomposition, the phase can be extracted from each path. On the other hand, it cannot be used for the calculation of TOF as it contains some unknown phase offset caused by ADC.

\subsection{PCA-based feature extraction}

Assuming that our system has $N$ transmitting antennas and $R$ receiving antennas. In line with the phase decomposition method, the dimension of a vector is $N^{*} R^{*} L=p$ where $L$ denotes the assumed number of paths. As each location consists of $T$ packets, we have $T p$-dimensional vector, and the size of a location matrix $\mathbf{V}$ is $T * P$ which may be a very high dimension for real time localization. To reduce the dimension and yield the robust feature, principal component analysis (PCA) is conducted for the location matrix. For the high dimension data, PCA selects the greatest variance dimension data as the principal components.

For the location matrix $\mathbf{V}$, we first calculate the median vector of the matrix and subtract it from each row to realize the mean-centered value. Set $\mathbf{M}$ and $\boldsymbol{\sigma}$ as the mean-centered matrix and the convenience matrix of $\mathbf{M}$, respectively. Assuming that $\lambda_{i}$ denotes the eigenvalue of $\boldsymbol{\sigma}$, and $x_{i}$ is eigenvector corresponding to eigenvalues. Thus, there is a vector $x_{i}$ which satisfies $\sigma x_{i}=\lambda_{i} x_{i}$. Let T eigenvectors and eigenvalue form matrix $\Phi$ and $\Lambda$ :

$$
\begin{aligned}
& \mathbf{\Phi}=\left[\begin{array}{llll}
x_{1} & x_{2} & \cdots & x_{T}
\end{array}\right] \\
& \boldsymbol{\Lambda}=\operatorname{diag}\left(\begin{array}{llll}
\lambda_{1} & \lambda_{2} & \cdots & \lambda_{T}
\end{array}\right)
\end{aligned}
$$

By normalizing every eigenvector to unit magnitude and orthogonal with each other, it is yielded that

$$
\begin{gathered}
\boldsymbol{\Phi}^{\prime} \boldsymbol{\Phi}=\boldsymbol{\Phi} \boldsymbol{\Phi}^{\prime}=\mathbf{I} \\
\boldsymbol{\sigma}=\boldsymbol{\Phi} \boldsymbol{\Lambda} \boldsymbol{\Phi}^{\prime}
\end{gathered}
$$

With $r$ largest eigenvalues of $\boldsymbol{\sigma}, T-r$ projection matrix is yielded:

$$
\boldsymbol{\Phi}=\left[\begin{array}{llll}
x_{1}^{p} & x_{2}^{p} & \cdots & x_{r}^{p}
\end{array}\right]
$$

For each location, we project the location matrix into a corresponding matrix as a fingerprint matrix:

$$
\mathbf{F}=\mathbf{M} \Phi_{p}
$$

\section{Experiment implementation and performance metrics}

5.1 Experimental equipment and scenarios

Our system is evaluated in the laboratory, meeting room, and corridor as shown in Fig. 5. The D-Link dir-859 wireless APs and the SONY laptops mounted WiFi Wireless Link 5300 Cards serve as transmitters and

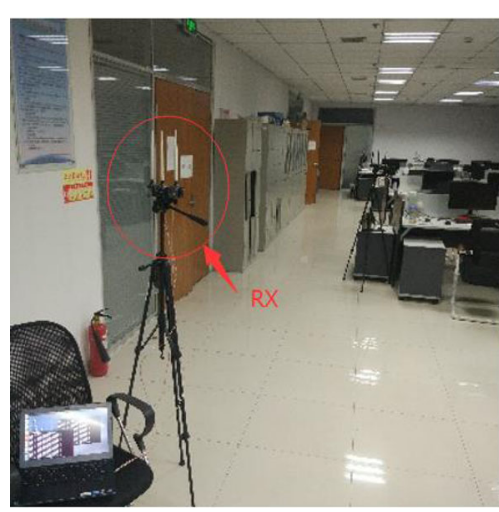

(1) Laboratory

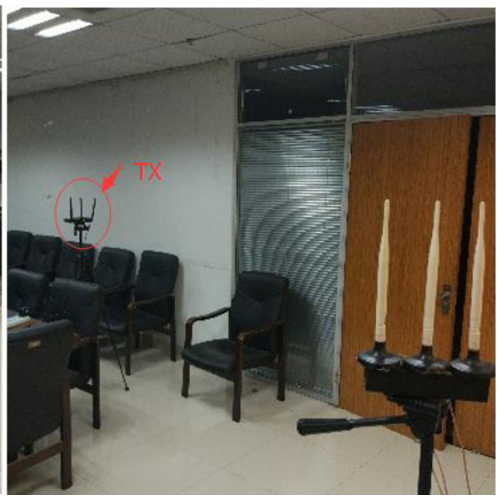

(2) Meeting room

Fig. 5 Experimental scenarios 
receivers, respectively. The APs operation in IEEE $802.11 \mathrm{n}$ mode has three antennas. In some places close to AP, the transmitting antenna and receiving antenna are very close, the signals from different antennas attenuating very similarly, causing the AP to actively merge similar channels. In this case, we are unable to obtain the CSI of all three antennas and the channel merging process is controlled by internal program of an AP that could not be intervened. Thereby, we use two of the three antennas as transmitting antennas.

We select the three experimental scenarios due to different floor areas and mobility. The scenarios can be categorized into static and dynamic based on the mobility, which depends on the number of people present and the frequency of their mobility during experimentation. In the meeting room, it is almost static with one people sitting at their place most of the time during experimentation. In contrast, the laboratory is fully occupied with the people at every desk location with the frequent mobility such as moving in and out of the place, therefore it can be a typical dynamic environment. Similar with the laboratory, corridor also can be seen as a dynamic environment since people frequently walk by.

In training phase, the areas fall into many cells with the size of about $0.6 \mathrm{~m} \times 0.6 \mathrm{~m}$ and label each cell with a number. At each cell, we collect 1000 packets from each AP. During the estimation phase, some cells are randomly selected, and 200 packets are gained at each cell. The layout and major parameters of three scenarios are shown in Fig. 6 and Table 1, respectively.

\subsection{Performance metrics}

Two metrics are used to evaluate the performance of our indoor localization system.

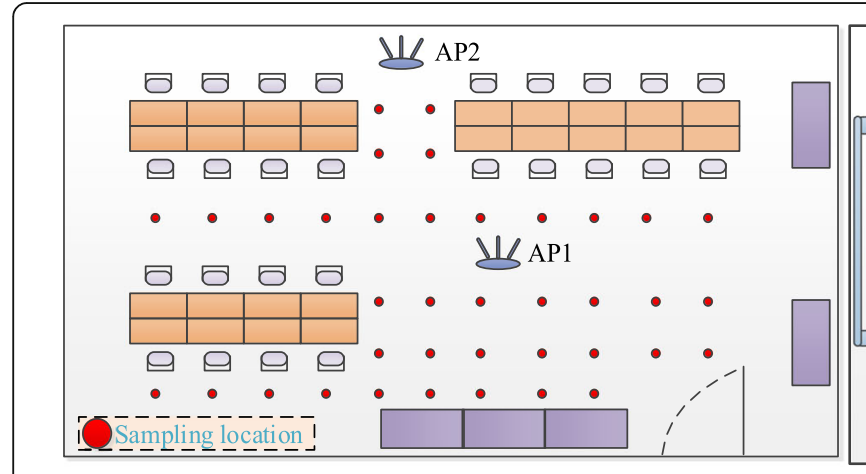

(1) Laboratory

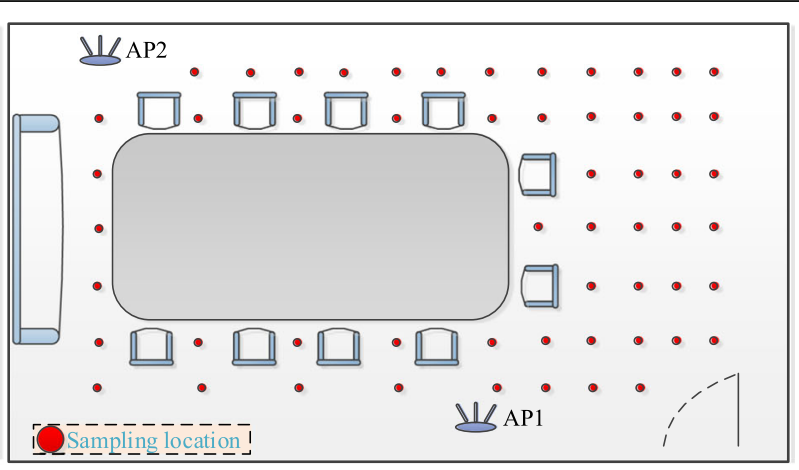

(2) Meeting room

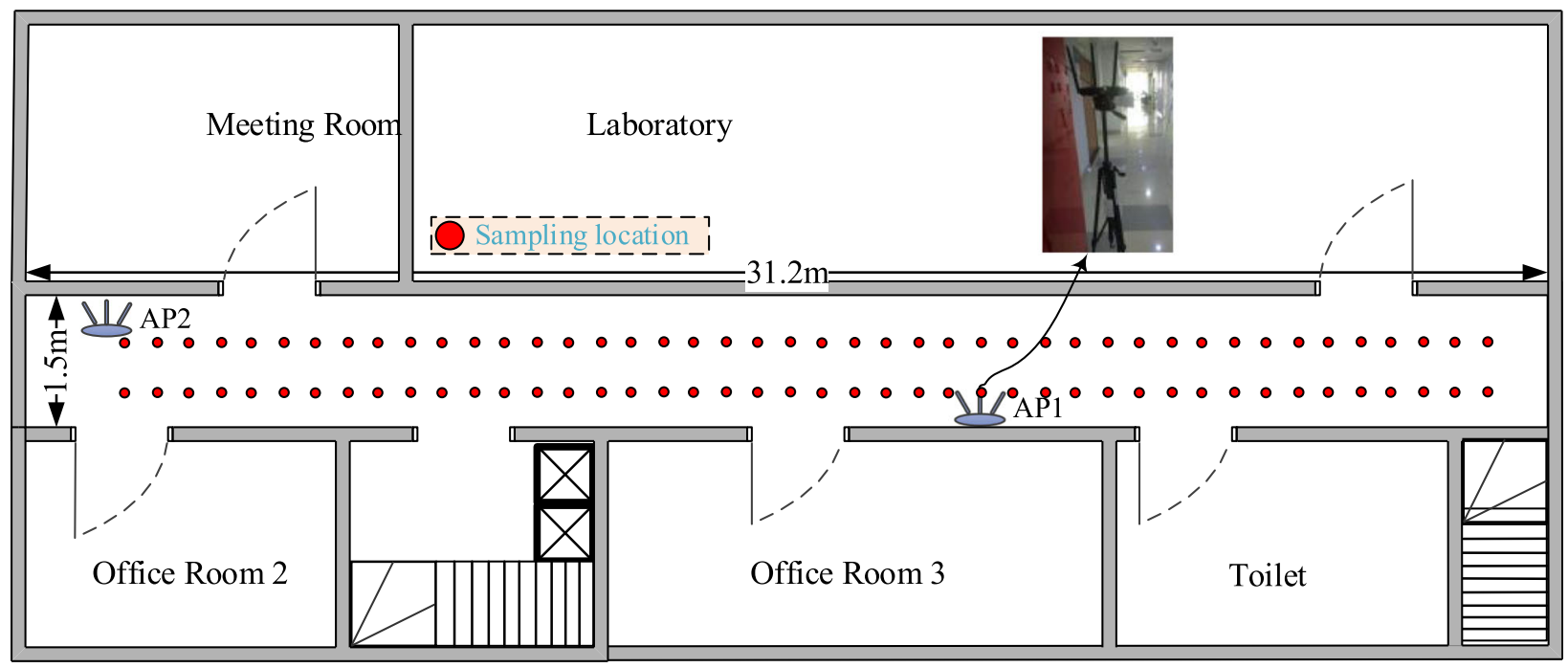

(3) Corridor

Fig. 6 Floor-plan of the three scenarios. The red points denote the sampling location. At each location, we collect 1000 packets from each AP 
Table 1 Main parameters of the scenarios

\begin{tabular}{llll}
\hline Parameters & Laboratory & Meeting room & Corridor \\
\hline Size & $23.6 \mathrm{~m} \times 5.8 \mathrm{~m}$ & $7.2 \mathrm{~m} \times 5.8 \mathrm{~m}$ & $31.2 \mathrm{~m} \times 1.5 \mathrm{~m}$ \\
Access points & 2 & 2 & 2 \\
Cells & 81 & 42 & 88 \\
\hline
\end{tabular}

\subsubsection{Cell estimation accuracy}

The cell estimation accuracy (CEA) suggests the ratio of the number of correctly estimated testing cells to all cell locations during the estimation phase, which is calculated as follows:

$$
\mathrm{CEA}=\frac{1}{N} \sum_{i=1}^{N} I\left(y_{i}=\hat{y}_{i}\right)
$$

where $N$ denotes the number of total testing cells.

\subsubsection{Median distance error}

The estimation phase may suggest that some testing cells are being misclassified. Given such circumstances, the average distance between the center points of the estimated cells and true cells are calculated, which is termed as median distance error. Formally, the median distance error is given by

$$
\operatorname{MDE}=\frac{1}{N} \sum_{i=1}^{N}\left\|y_{i}-\hat{y}_{i}\right\|_{2}
$$

where $\|\cdot\|_{2}$ denotes the L2 norm.

Combining with the size of cell size, the CEA could be used to express the precision of positioning and the median distance error (MDE) is utilized to represent the overall performance of positioning system.

\subsection{Overall performance}

The most machine learning algorithms can be categorized into deterministic and probabilistic algorithms, so we selected KNN, SVM, and Bayes as matching algorithms. KNN and SVM are deterministic algorithms which use the distance between a test fingerprint and each training fingerprint from the fingerprint database to estimate the test location. Bayes algorithm was a probabilistic algorithm that estimates the test location by maximizing the posterior probability.

Three fingerprint matching algorithms are used to examine the overall performance of our system in the three scenarios with only one AP. The cell estimation accuracy and median distance error in three scenarios are shown in Fig. 7. It is observed from Fig. 7 that the CEA in laboratory is higher than that in the meeting room, while the MDE is lower than that in the meeting room and among all scenarios, the corridor also reaches the maximum MDE and the minimum CEA. This is primarily because the furniture, the number of people, and the frequency of their mobility in the meeting room are less than those in the laboratory. Thus, the multipath effect is small during our experiment. In comparison with the other matching algorithms, SVM reaches the best cell estimation accuracy and the lowest median distance error in both scenarios. The overall performance in the two different environments is summarized in Table 2.
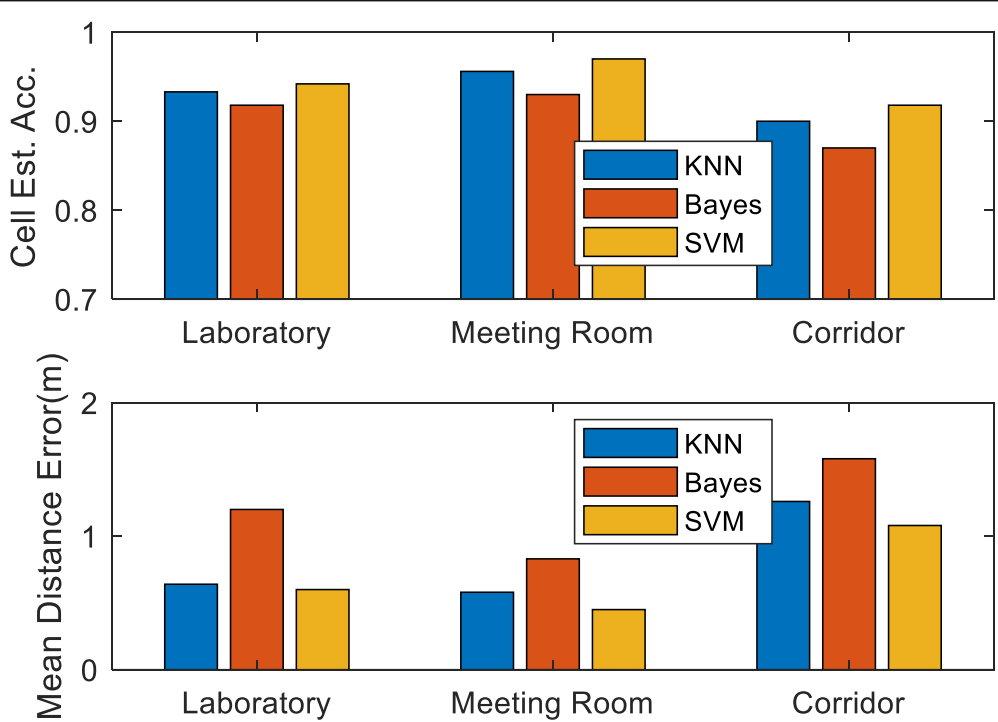

Fig. 7 Cell estimation accuracy and median distance error in three scenarios. In the top figure blue, orange and yellow bar represent the cell estimation accuracy for using KNN, Bayes, and SVM as the match algorithm in the laboratory and meeting room, respectively. In the bottom figure, blue, orange, and yellow bar represent the median distance error for using KNN, Bayes, and SVM as the match algorithm in the laboratory, in the meeting room and in the corridor, respectively 
Table 2 Overall performance

\begin{tabular}{lllll}
\hline Scenario & Performance metric & KNN & Bayes & SVM \\
\hline Laboratory & CEA & 0.933 & 0.918 & 0.942 \\
& MDE $(\mathrm{m})$ & 0.64 & 1.20 & 0.60 \\
Meeting room & CEA & 0.956 & 0.93 & 0.97 \\
& MDE $(\mathrm{m})$ & 0.58 & 0.83 & 0.45 \\
\hline
\end{tabular}

\subsection{Impact of the number of decomposition path}

As the number of specific paths in the environment cannot be accurately obtained, we can only intuitively assume the number of paths in the environment. We investigate the impact of the number of decomposition paths, as shown in Fig. 8. It is observed that more decomposition paths do not result in higher positioning accuracy. In contrast, due to the noise in the indoor environment, considerable decomposition path may make it difficult for differentiation of each fingerprint, as the fingerprint dimension increasing more noise is introduced to the fingerprint. We believe that 2-3 decomposition paths are appropriate in a scenario where the multipath effect is not obvious, and 4-5 decomposition paths are appropriate in a multipath effect environment.

\subsection{Impact of size of training and estimation samples}

We have evaluated four combinations: $1 \mathrm{k} / 200,500 / 100$, 200/50, and 50/50. Figure 9 presents the MDE of four combinations. Bayes algorithm needs to calculate the prior probabilities, and if there is not enough data for calculating the prior probabilities, the location accuracy would decrease Moreover, in Bayesian algorithm, it is assumed that the features are independently and identically distributed; however, this assumption is often not valid in practice. Therefore, it can be seen from the Fig. 9, on the condition of small size of training and estimation samples Bayes achieves the highest MDE compared to KNN and SVM.

KNN calculates the Euclidean distance between the features of test location and the features in the fingerprint database to estimate location. The same as Bayes algorithm, it also needs a large amount data to improve the location accuracy. SVM is different from KNN, as it can build the model for classification only using a few samples. Additionally, SVM is good at dealing with high-dimensional and non-linear data.

The SVM approach performs the best in all of combinations as shown in Table 3. The best accuracy is $0.6 \mathrm{~m}$ in the laboratory and $0.45 \mathrm{~m}$ in the meeting room. They are both reached under the combination of $1 \mathrm{~K} / 200$ with SVM algorithm. In comparison with the other two algorithms, Bayes algorithm needs more data to achieve high accuracy as it calculates the prior and posterior probabilities. In brief, a larger amount of data improves the estimation of the data characteristics and is conducive to building a more accurate model to improve the positioning accuracy.
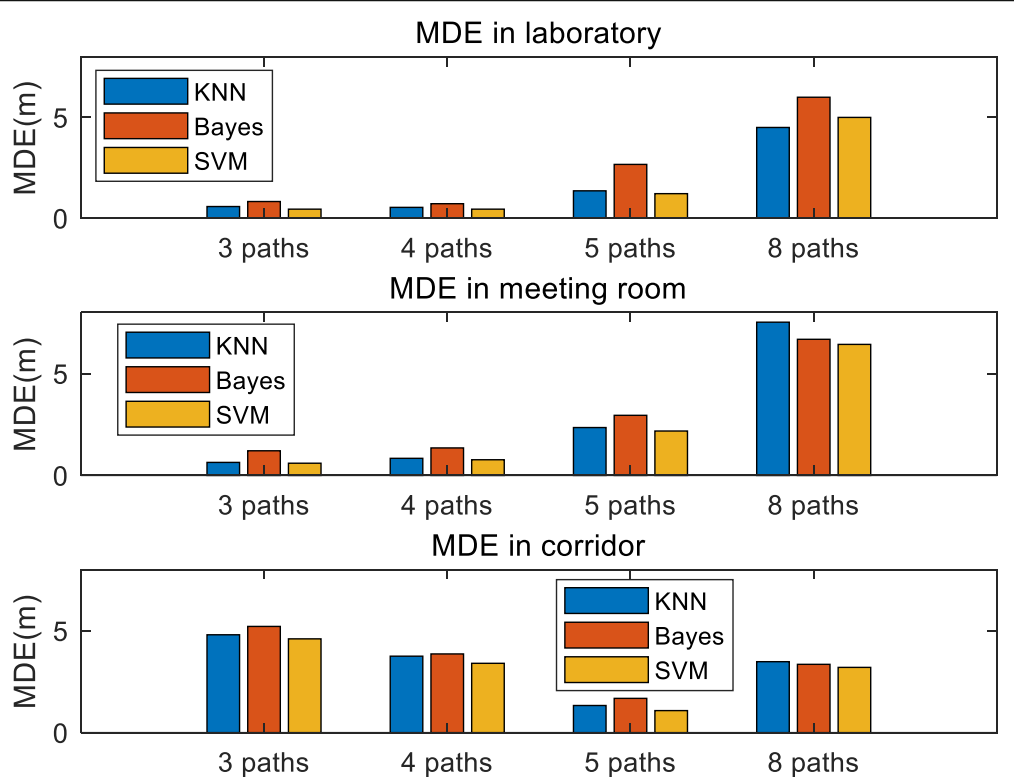

Fig. $8 \mathrm{MDE}$ for different number of decomposition path. In the top figure, blue, orange, and yellow bar represent the median distance error of different decomposition path by using KNN, Bayes, and SVM as the match algorithm in the laboratory, respectively. In the middle figure, blue, orange, and yellow bar represent the median distance error of different decomposition path by using KNN, Bayes, and SVM as the match algorithm in the meeting room, respectively. In the bottom figure, blue, orange, and yellow bar represent the median distance error of different decomposition path by using KNN, Bayes, and SVM as the match algorithm in the corridor, respectively 

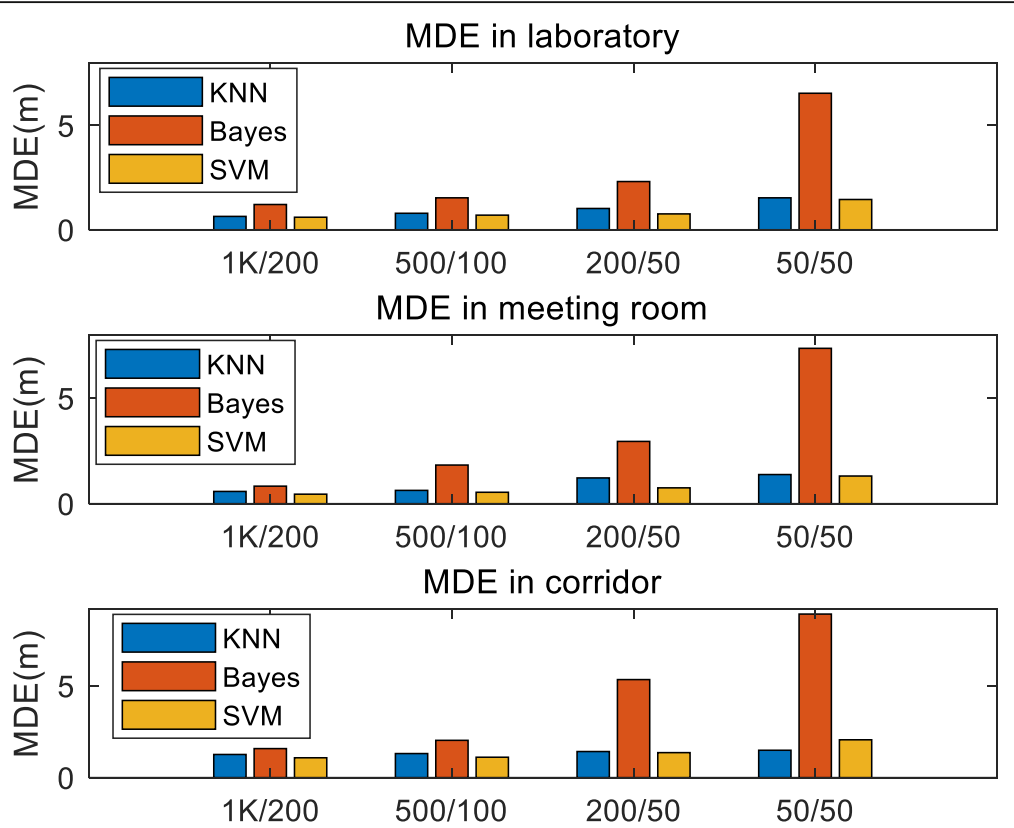

Fig. $9 \mathrm{MDE}$ for different size of training and estimation samples. In the top figure, blue, orange, and yellow bar represent the median distance error of different size of training and estimation samples by using KNN, Bayes, and SVM as the match algorithm in the laboratory, respectively. In the middle figure, blue, orange, and yellow bar represent the median distance error of different size of training and estimation samples by using KNN, Bayes, and SVM as the match algorithm in the meeting room, respectively. In the bottom figure, blue, orange, and yellow bar represent the median distance error of different size of training and estimation samples by using KNN, Bayes, and SVM as the match algorithm in the corridor, respectively

\subsection{Impact of the number of APs}

Although our system only uses one AP to reach accurate localization, the number of APs is also a crucial factor for localization. In all scenarios, two APs are used to investigate the impact for positional accuracy. The MDE for different numbers of APs is demonstrated in Fig. 10. It is observed that adding an AP will only reduce the MDE by about $0.05 \mathrm{~m}$ when

Table 3 MDE for different sizes of training and estimation samples

\begin{tabular}{lllll}
\hline Scenario & Sample size & KNN $(\mathrm{m})$ & Bayes $(\mathrm{m})$ & SVM $(\mathrm{m})$ \\
\hline Laboratory & $1 \mathrm{~K} / 200$ & 0.64 & 1.21 & 0.6 \\
& $500 / 100$ & 0.79 & 1.53 & 0.7 \\
& $200 / 50$ & 1.02 & 2.31 & 0.76 \\
Meeting room & $1 \mathrm{~K} / 200$ & 0.58 & 0.83 & 0.45 \\
& $500 / 100$ & 0.63 & 1.83 & 0.54 \\
& $200 / 50$ & 1.22 & 2.95 & 0.75 \\
& $50 / 50$ & 1.38 & 7.36 & 1.31 \\
Corridor & $1 \mathrm{~K} / 200$ & 1.26 & 1.58 & 1.08 \\
& $500 / 100$ & 1.31 & 2.03 & 1.11 \\
& $200 / 50$ & 1.42 & 5.34 & 1.36 \\
& $50 / 50$ & 1.49 & 8.91 & 2.06 \\
\hline
\end{tabular}

KNN and SVM approaches are used for localization. This level of reduction is inconsequential in some application scenarios. However, it would be very useful for Bayes to increase the accuracy whose MDE drop from 1.21 to $1.08 \mathrm{~m}$ in the laboratory and from 0.83 to $0.63 \mathrm{~m}$ in the meeting room, respectively.

\subsection{Impact of the size of cell}

The size of cell is also an important factor that affects the positon accuracy. We conduct experiments in laboratory, meeting room, and corridor respectively to analyze the impact of the sizes of cell. We select three size of cell which are $0.3 \mathrm{~m} \times 0.3 \mathrm{~m}, 0.6 \mathrm{~m} \times 0.6 \mathrm{~m}$, and $1.2 \mathrm{~m} \times 1.2 \mathrm{~m}$. The results are shown in Fig. 11. As can be seen from the figure, the size of $0.6 \mathrm{~m} \times 0.6 \mathrm{~m}$ reaches the minimum mean distance error. Since the variance of the feature in a fingerprint is not small enough compared to the distance between two adjacent cells, if the size is too small, the features of the fingerprint would be very similar to that of the fingerprint of the adjacent cell in feature space, which would make it difficult to distinguish. Hence, too small size would not help to improve positioning accuracy, but would only increase the labor and time during building the fingerprint database. On the other hand, if the size is too big, the measurement scale would be more extensive which would not be beneficial to improve the position accuracy, too. 

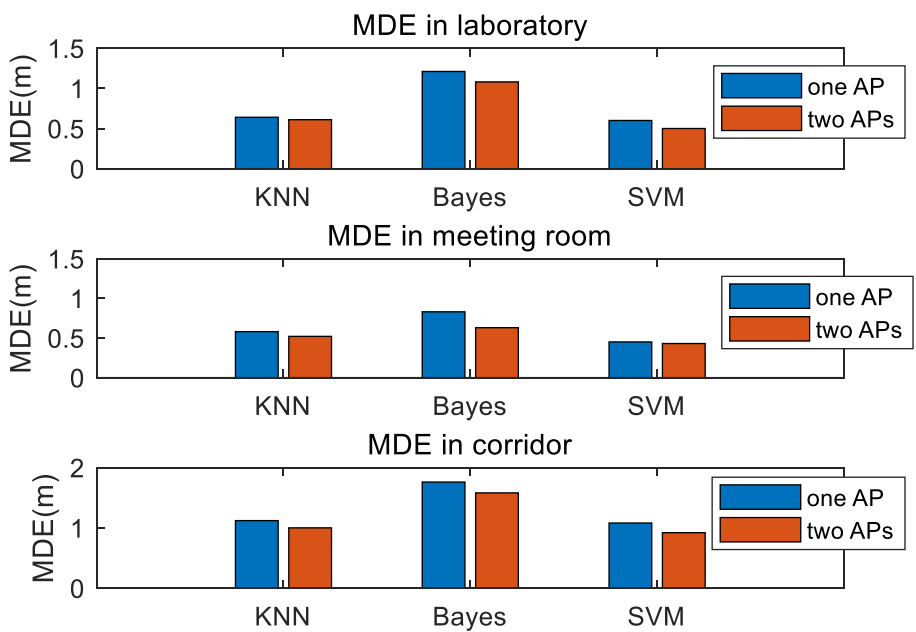

Fig. $10 \mathrm{MDE}$ for different number of APs. In the top figure, blue and orange represent the median distance error of different match algorithm under the one AP and two APs conditions in the laboratory, respectively. In the middle figure, blue and orange represent the median distance error of different match algorithm under the one AP and two APs conditions in the meeting room, respectively. In the bottom figure, blue and orange represent the median distance error of different match algorithm under the one AP and two APs conditions in the corridor, respectively

\subsection{Comparison with other localization system}

Our localization system is compared with two fingerprint-based systems, i.e., a RSSI-based system (RADAR) [33] and a CSI-based system (CSI-MIMO) [20] in the three environments. In the experiments, the RSSI used in the RADAR system is the mean signal strength of the three receiving antennas and in the
CSI-based system, we use the same CSI data as that in our system. KNN approach is introduced to all the three systems for matching fingerprints. The performance of the three systems under the conditions of one AP and two APs are shown in Fig. 11.

Since an AP only provides one RSSI, the RADAR system lacks features for localization whose $\mathrm{MDE}$ is

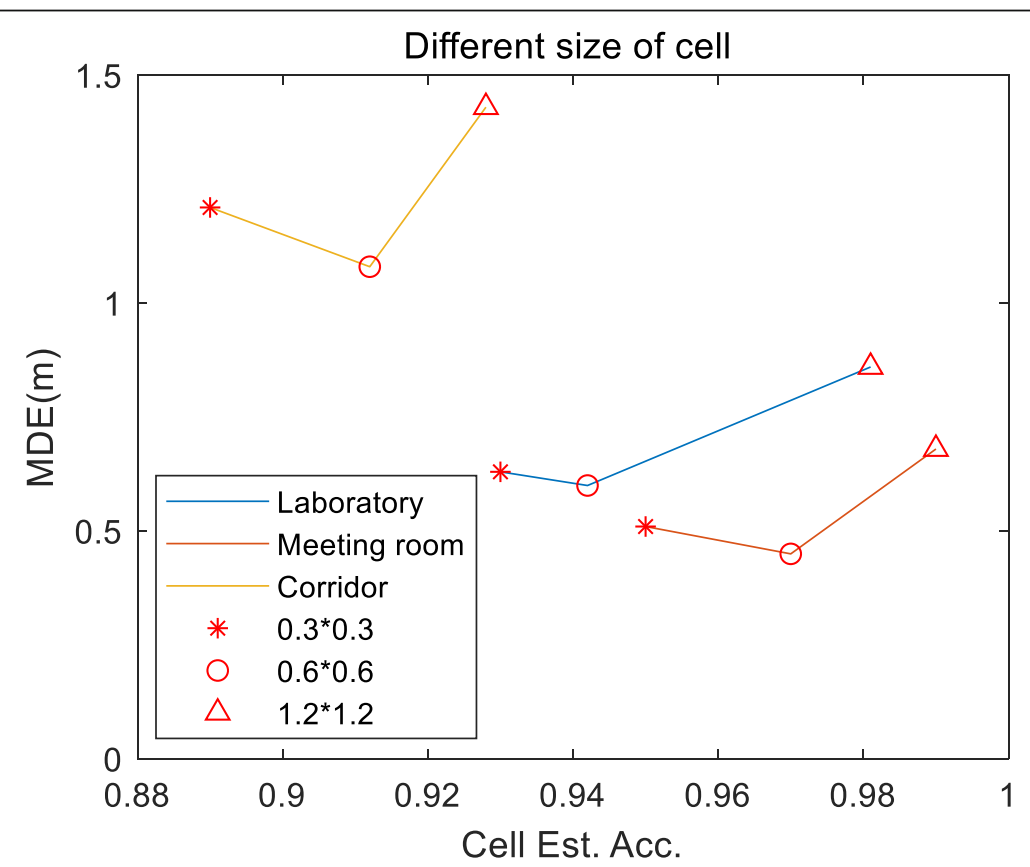

Fig. 11 Different size of cell. In the figure, blue, orange, and yellow line represents the experiment scenarios. Blue represents the experiment done in the laboratory, orange line represents the experiment done in the meeting room and yellow line represents the experiment done in the corridor. Star represents the size of cell is $0.3 \mathrm{~m} \times 0.3 \mathrm{~m}$, circular represents the size of cell is $0.6 \mathrm{~m} \times 0.6 \mathrm{~m}$, and triangle represents the size of cell is $1.2 \mathrm{~m} \times 1.2 \mathrm{~m}$ 


\section{MDE in One AP}
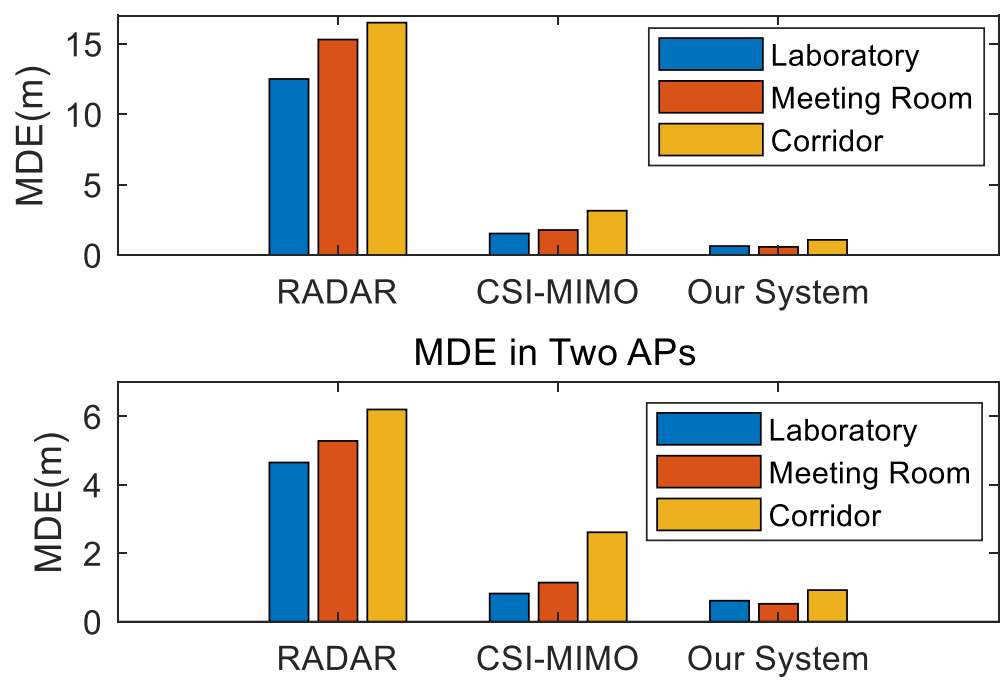

Fig. 12 Comparison with other localization system. In the top figure, blue, red, and orange bar represent the median distance error of different localization system with one AP in the laboratory, meeting room, and the corridor, respectively. In the top figure, blue, red, and orange bar represent the median distance error of different localization system with two AP in the laboratory, meeting room and the corridor, respectively

$15.3 \mathrm{~m}$ in laboratory, $12.5 \mathrm{~m}$ in the meeting room, and $16.5 \mathrm{~m}$ in the corridor, respectively. Two APs contribute to reducing the MDE for RADAR, the MDE decreases significantly in all three scenarios. Similar to RADAR, more APs are also conducive to improving the localization accuracy for our system. With two APs, the MDE of our system is $0.61 \mathrm{~m}$ in the laboratory, which is better than $0.82 \mathrm{~m}$ reached by CSI-MIMO. In terms of one AP, the advantage of our system is apparent, reaching $0.64 \mathrm{~m}$ in the laboratory, $0.58 \mathrm{~m}$ in the meeting room, and $1.12 \mathrm{~m}$ in the corridor. Moreover, this is superior over $1.53 \mathrm{~m}, 1.78 \mathrm{~m}$, and $3.15 \mathrm{~m}$ of CSI-MIMO system, respectively (Fig. 12).

\section{Conclusion}

In this study, a fingerprint-based localization system is presented that only using a single AP. We use the phase information for fingerprinting localization and a linear transformation algorithm to eliminate the noise in the phase. A new phase decomposition method is proposed to acquire the phase information of multipath, and a PCA-based algorithm is used to extract features for the generation of a fingerprint. The performance in the laboratory, the meeting room, and the corridor is validated. The results reveal a minimum mean distance error of $0.6 \mathrm{~m}$ in the laboratory, $0.45 \mathrm{~m}$ in the meeting room, and $1.08 \mathrm{~m}$ in the corridor. We also analyze the impact factors on the localization accuracy of our system and compare our system with a RSSI-based and a CSI-based fingerprint localization system. The results demonstrate that our system outperforms the other two systems.
Our future work will focus on more complex scenarios where multiple sensing elements are used to obtain more exact information for localization. Research is ongoing to verify whether the current system can also be extended to a multi-level floor.

\section{Abbreviations}

CSI: Channel state information; LBS: Location-based service; MIMO: Multiple input multiple output; OFDM: Orthogonal frequency division multiplexing; RSSI: Received signal strength indication

\section{Acknowledgements}

The authors would like to thank the reviewers for their helpful advice.

Funding

This work is supported by the National key research and development plan under Grant (No: 2017YFC0804401).

Availability of data and materials

The data used to support the findings of this study can be obtained by emailing the corresponding author.

Authors' contributions

All authors read and approved the final manuscript.

\section{Competing interests}

The authors declare that they have no conflict of interests.

\section{Publisher's Note}

Springer Nature remains neutral with regard to jurisdictional claims in published maps and institutional affiliations.

\section{Author details}

'School of Information and Control Engineering, China University of Mining and Technology, Xuzhou 221000, China. ${ }^{2}$ loT Perception Mine Research Center, China University of Mining and Technology, Xuzhou 221000, China. ${ }^{3}$ National and Local Joint Engineering Laboratory of Internet Application Technology on Mine, Xuzhou 221008, Jiangsu, China. 


\section{Received: 27 September 2018 Accepted: 14 February 2019} Published online: 28 February 2019

\section{References}

1. G.A. Amagsila, M.E. Cabuhat, J.E. Tigbayan, et al., A framework for mobile application of flood alert monitoring system for vehicle users using Arduino device $[\mathrm{C}] / /$ humanoid, nanotechnology, information technology, communication and control, environment and management (HNICEM), 2017 IEEE 9th international conference on. IEEE, 1-6 (2017)

2. F.A. Milaat, H. Liu, Decentralized detection of GPS spoofing in vehicular ad hoc networks [J]. IEEE Commun. Lett. 99, 1-1 (2018)

3. S. He, S.H.G. Chan, Wi-fi fingerprint-based indoor positioning: recent advances and comparisons [J]. IEEE Communications Surveys \& Tutorials 18(1), 466-490 (2017)

4. K. QIAN et al., Enabling phased array signal processing for mobile WiFi devices. IEEE Trans. Mob. Comput. 17(8), 1820-1833 (2018)

5. M.O.G.H.T.A.D.A.I.E.E. Vahideh, G. DEMPSTER Andrew, Determining the best vector distance measure for use in location fingerprinting. Pervasive and Mobile Computing 23, 59-79 (2015)

6. S. He, S.H.G. Chan, L. Yu, et al., SLAC: calibration-free pedometer-fingerprint fusion for indoor localization [J]. IEEE Trans. Mob. Comput. 17(5), 1176-1189 (2018)

7. S. He, W. Lin, S.H.G. Chan, Indoor localization and automatic fingerprint update with altered AP signals [J]. IEEE Trans. Mob. Comput. 16(7), 1897$1910(2017)$

8. X. Tian, M. Wang, W. Li, et al., Improve accuracy of fingerprinting localization with temporal correlation of the RSS [J]. IEEE Trans. Mob. Comput. 17(1), 113-126 (2018)

9. W. Su, E. Liu, A.M. Calveras Augé, et al., Design and realization of precise indoor localization mechanism for Wi-Fi devices [C]//KSII transactions on internet and. Inf. Syst. 10(12), 5422-5441 (2016)

10. M. Youssef, A. Agrawala, The Horus location determination system [J]. Wirel. Netw 14(3), 357-374 (2008)

11. Z. Yang, Z. Zhou, Y. Liu, From RSSI to CSI:Indoor localization via channel response [J]. ACM Comput. Surv. 46(2), 1-32 (2013)

12. S. Yiu, M. Dashti, H. Claussen, et al., Wireless RSSI fingerprinting localization [J]. Signal Process. 131, 235-244 (2017)

13. A.K.M.M. Hossain, Y. Jin, W.S. Soh, et al., SSD: a robust RF location fingerprint addressing mobile devices' heterogeneity [J]. IEEE Trans. Mob. Comput. 12(1), 65-77 (2013)

14. "802.11n-2009 - IEEE Standard for Information technology-- Local and metropolitan area networks-- Specific requirements-- Part 11: Wireless LAN Medium Access Control (MAC) and Physical Layer (PHY) Specifications Amendment 5: Enhancements for Higher Throughput," 2009, 1-565

15. S. Sen, J. Lee, K.H. Kim, et al., Avoiding multipath to revive inbuilding WiFi localization [C]// proceeding of the, international conference on Mobile systems, applications, and services. ACM, 249-262 (2013)

16. D. Halperin, W. Hu, A. Sheth, et al., Tool release: gathering $802.11 \mathrm{n}$ traces with channel state information [J]. ACM SIGCOMM Computer Communication Review 41(1), 53-53 (2011)

17. R. Zhou, J. Chen, X. Lu, et al., CSI fingerprinting with SVM regression to achieve device-free passive localization [C]// IEEE, international symposium on a world of wireless, Mobile and multimedia networks. IEEE, 1-9 (2017)

18. Z. Tian, Z. Li, M. Zhou, et al., Sub-meter localization using CSI from commodity Wi-Fi devices [J]. Sensors 16(10), 1664 (2016)

19. X. Wang, L. Gao, S. Mao, et al., DeepFi: deep learning for indoor fingerprinting using channel state information $[\mathrm{C}] / /$ wireless communications and NETWORKING conference. IEEE, 1666-1671 (2015)

20. Y. Chapre, A. Ignjatovic, A. Seneviratne, et al., CSI-MIMO: An efficient Wi-fi fingerprinting using channel state information with MIMO [J]. Pervasive \& Mobile Computing 23, 89-103 (2015)

21. J. Wang, H. Jiang, J. Xiong, et al., LiFS: low human-effort, device-free localization with fine-grained subcarrier information [C]// international conference on Mobile computing and NETWORKING. ACM, 243-256 (2016)

22. K. Wu, J. Xiao, Y. Yi, et al., CSI-based indoor localization [J]. IEEE Transactions on Parallel \& Distributed Systems 24(7), 1300-1309 (2013)

23. J. Xiao, K. Wu, Y. Yi, et al., FIFS: Fine-grained indoor fingerprinting system $[C] / /$ international conference on computer communications and networks. IEEE, 1-7 (2012)
24. J. Xiong, K. Jamieson, ArrayTrack: A fine-grained indoor location system [C]// Usenix conference on networked systems design and implementation. USENIX Association, 71-84 (2013)

25. J. Xiao, K. Wu, Y. Yi, et al., Pilot: Passive device-free indoor localization using channel state information [C]//distributed computing systems (ICDCS), 2013 IEEE 33rd international conference on. IEEE, 236-245 (2013)

26. M. Seifeldin, A. Saeed, A.E. Kosba, et al., Nuzzer: a large-scale device-free passive localization system for wireless environments [J]. IEEE Trans. Mob. Comput. 12(7), 1321-1334 (2013)

27. J. Wang, L. Zhang, X. Wang, et al., A novel CSI pre-processing scheme for device-free localization indoors [C]//proceedings of the eighth wireless of the students, by the students, and for the students workshop. ACM, 6-8 (2016)

28. G. Yang, WiLocus: CSI based human tracking system in indoor environment [C]//measuring technology and mechatronics automation (ICMTMA), 2016 eighth international conference on. IEEE, 915-918 (2016)

29. J.W. Yang, G. Cho, Utilizing CSI to improve distance estimation precision in the indoor environment [J]. International Journal of Software Engineering and Its Applications 9(3), 49-56 (2015)

30. S. Shi, S. Sigg, Y. Ji, Probabilistic fingerprinting based passive device-free localization from channel state information [C]//vehicular technology conference (VTC spring), 2016 IEEE 83rd. IEEE, 1-5 (2016)

31. R. Zhou, J. Chen, X. Lu, et al., CSI fingerprinting with SVM regression to achieve device-free passive localization [C]//a world of wireless, mobile and multimedia networks (WoWMoM), 2017 IEEE 18th international symposium on. IEEE, 1-9 (2017)

32. X. Li, S. Li, D. Zhang, et al., Dynamic-music: accurate device-free indoor localization [C]//proceedings of the 2016 ACM international joint conference on pervasive and ubiquitous computing. ACM, 196-207 (2016)

33. P. Bahl, V.N. Padmanabhan, RADAR: an in-building RF-based user location and tracking system [C]//INFOCOM 2000. Nineteenth annual joint conference of the IEEE computer and communications societies. Proceedings. IEEE. 2, 775-784 (2000)

34. X. Wang, L. Gao, S. Mao, CSI phase fingerprinting for indoor localization with a deep learning approach [J]. IEEE Internet Things J. 3(6), 1113-1123 (2016)

35. J. Xiong, K. Sundaresan, K. Jamieson, Tonetrack: Leveraging frequency-agile radios for time-based indoor wireless localization $[C] / /$ proceedings of the 21st annual international conference on Mobile computing and networking. ACM, 537-549 (2015)

36. D. Vasisht, S. Kumar, D. Katabi, Decimeter-level localization with a single WiFi access point [C]//NSDI, vol 16 (2016), pp. 165-178

37. S. Cai, W. Liao, C. Luo, et al., CRIL: an efficient online adaptive indoor localization system [J]. IEEE Trans. Veh. Technol. 66(5), 4148-4160 (2017)

38. D. Halperin, W. Hu, A. Sheth, et al., Predictable 802.11 packet delivery from wireless channel measurements [C]//ACM SIGCOMM computer communication review. ACM 40(4), 159-170 (2010)

39. D. Tse, P. Viswanath, Fundamentals of wireless communication [M] (Cambridge university press, 2005)

40. C. Shen, X. Bao, J. Tan, et al., Two noise-robust axial scanning multi-image phase retrieval algorithms based on Pauta criterion and smoothness constraint [J]. Opt. Express 25(14), 16235-16249 (2017)

41. S. Sen, B. Radunovic, R.R. Choudhury, et al., You are facing the Mona Lisa: spot localization using PHY layer information [C]//proceedings of the 10th international conference on Mobile systems, applications, and services. ACM, 183-196 (2012)

42. M.K. Ng, R.H. Chan, W.C. Tang, A fast algorithm for deblurring models with Neumann boundary conditions [J]. SIAM J. Sci. Comput. 21(3), 851-866 (1999)

43. D.L. Boley, F.T. Luk, D. Vandevoorde, A general vandermonde factorization of a Hankel matrix [C]//Int'l Lin. Alg. Soc.(ILAS) Symp. on Fast Algorithms for Control, Signals and Image Processing (1997) 